\title{
Assessing the Calibration of Dichotomous Outcome Models with the Calibration Belt
}

\author{
Giovanni Nattino
}

The Ohio Colleges of Medicine Government Resource Center

The Ohio State University

Stata Conference - July 19, 2018 


\section{Background: Logistic Regression}

- Most popular family of models for binary outcomes $(Y=1$ or $Y=0)$;

- Models $\operatorname{Pr}(Y=1)$, probability of "success" or "event";

- Given predictors $X_{1}, \ldots, X_{p}$, the model is

$$
\operatorname{logit}\{\operatorname{Pr}(Y=1)\}=\beta_{0}+\beta_{1} X_{1}+\ldots+\beta_{p} X_{p},
$$

where $\operatorname{logit}(\pi)=\log (\pi /(1-\pi))$.

- Does my model fit the data well? 


\section{Goodness of Fit of Logistic Regression Models}

Let $\hat{\pi}$ be the model's estimate of $\operatorname{Pr}(Y=1)$ for a given subject.

Two measures of goodness of fit:

- Discrimination

- Do subjects with $Y=1$ have higher $\hat{\pi}$ than subjects with $Y=0$ ?

- Evaluated with area under ROC curve.

- Calibration

- Does $\hat{\pi}$ estimate $\operatorname{Pr}(Y=1)$ accurately? 


\section{An Example: ICU Data}

- logit sta age can sysgp_4 typ locd

Iteration 0: $\quad$ log likelihood $=-100.08048$

Iteration 1: $\quad$ log likelihood $=-70.385527$

Iteration 2: $\quad$ log likelihood $=-67.395341$

Iteration 3: $\quad$ log likelihood $=-66.763511$

Iteration 4: $\quad \log$ likelihood $=-66.758491$

Iteration 5: $\quad$ log likelihood $=-66.758489$

Logistic regression

Number of obs=

200

LR chi2(5)

$=$

66.64

Prob > chi2

Log likelihood $=-66.758489$

Pseudo R2

$=$

0.0000

$=0.3330$

\begin{tabular}{r|rrrrrr}
\hline sta & Coef. & Std. Err. & z & P $>|z|$ & [95\% Conf. Interval] \\
\hline age & .040628 & .0128617 & 3.16 & 0.002 & .0154196 & .0658364 \\
can & 2.078751 & .8295749 & 2.51 & 0.012 & .4528141 & 3.704688 \\
sysgp_4 & -1.51115 & .7204683 & -2.10 & 0.036 & -2.923242 & -.0990585 \\
typ & 2.906679 & .9257469 & 3.14 & 0.002 & 1.092248 & 4.72111 \\
locd & 3.965535 & .9820316 & 4.04 & 0.000 & 2.040788 & 5.890281 \\
c cons & -6.680532 & 1.320663 & -5.06 & 0.000 & -9.268984 & -4.09208 \\
\hline
\end{tabular}




\section{An Example: ICU Data}

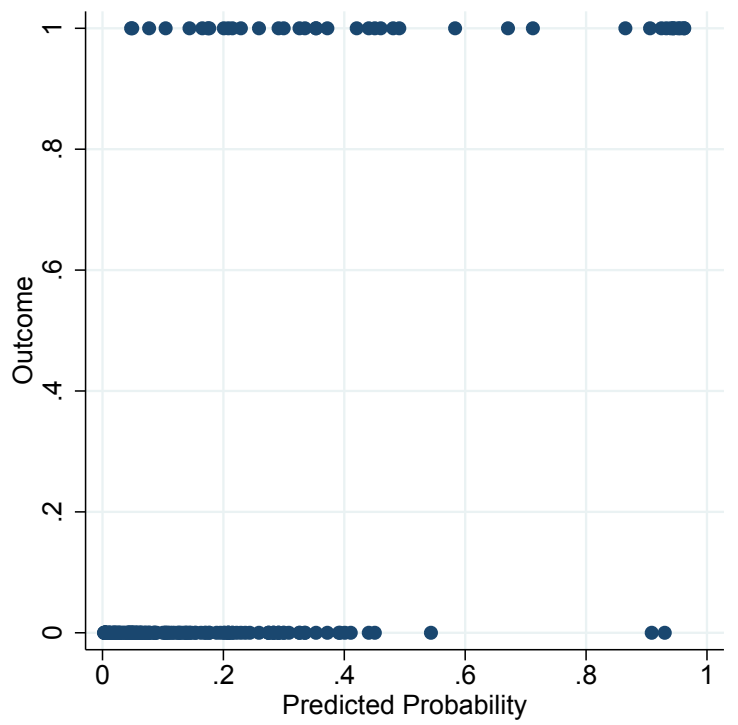




\section{An Example: ICU Data}

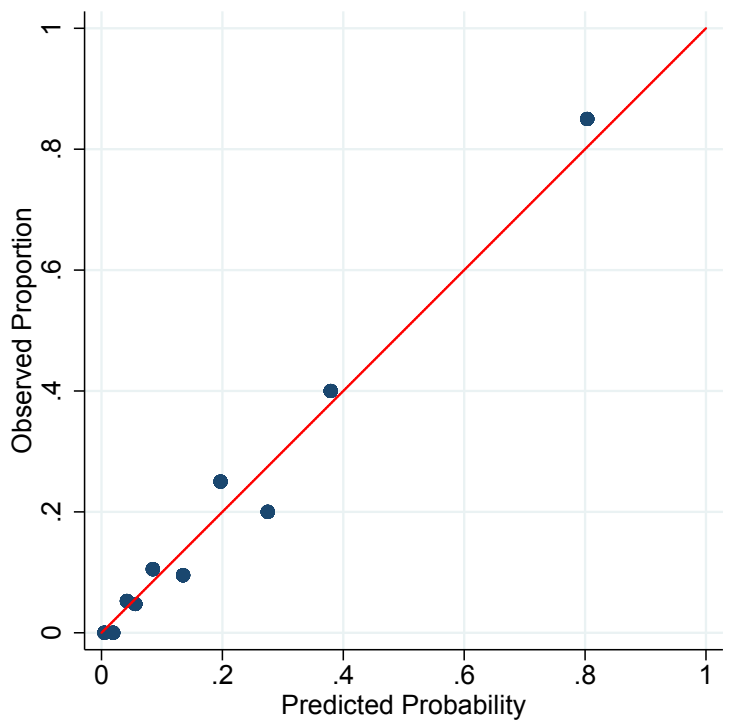




\section{The Hosmer-Lemeshow Test}

- Divide data into $G$ groups (usually, $G=10$ ).

- For each group, define:

- $O_{1 g}$ and $E_{1 g}$ : number of observed and expected events $(Y=1)$.

- $O_{0 g}$ and $E_{0 g}$ : number of observed and expected non-events $(Y=0)$.

- The Hosmer-Lemeshow statistic is:

$$
\widehat{C}=\sum_{g=1}^{G}\left[\frac{\left(O_{1 g}-E_{1 g}\right)^{2}}{E_{1 g}}+\frac{\left(O_{0 g}-E_{0 g}\right)^{2}}{E_{0 g}}\right]
$$

- Under the hypothesis of perfect fit, $\widehat{C} \sim \chi_{G-2}^{2}$.

- Problems:

- How many groups?

- Different $G$, different results.

Hosmer Jr, D. W., Lemeshow, S., Sturdivant, R. X. (2013). Applied logistic regression. 


\section{The Calibration Curve}

- Let $\widehat{g}=\operatorname{logit}(\widehat{\pi})$. What about fitting a new model:

$$
\operatorname{logit}\{P(Y=1)\}=\alpha_{0}+\alpha_{1} \widehat{g} \text {. }
$$

- If $\alpha_{0}=0$ and $\alpha_{1}=1$,

$$
\begin{gathered}
\operatorname{logit}\{P(Y=1)\}=0+1 \times \widehat{g}=\widehat{g} \\
\Downarrow \\
\operatorname{logit}\{P(Y=1)\}=\operatorname{logit}(\widehat{\pi}) \\
\Downarrow \\
P(Y=1)=\hat{\pi}
\end{gathered}
$$

If perfect fit, $\widehat{\alpha}_{0}=0$ and $\widehat{\alpha}_{1}=1$.

- Problems:

- Only for external validation of the model.

- Why linear relationship?

Cox, D. (1958). Two further applications of a model for a method of binary regression. Biometrika. 


\section{The Calibration Curve}

We assume a general polynomial relationship:

$$
\operatorname{logit}\{P(Y=1)\}=\alpha_{0}+\alpha_{1} \hat{g}+\alpha_{2} \hat{g}^{2}+\ldots+\alpha_{m} \hat{g}^{m} .
$$

$m ?$

- fixed too low $\Rightarrow$ too simplistic;

- fixed too high $\Rightarrow$ estimation of useless parameters;

Solution: Forward selection. 


\section{Example: ICU Data}

Selected polynomial is $m=2$ :

$$
\operatorname{logit}\{P(Y=1)\}=0.117+0.917 \hat{g}-0.076 \hat{g}^{2} .
$$

This defines the calibration curve

$$
P(Y=1)=\frac{e^{0.117+0.917 \operatorname{logit}(\hat{\pi})-0.076(\operatorname{logit}(\hat{\pi}))^{2}}}{1+e^{0.117+0.917 \operatorname{logit}(\hat{\pi})-0.076(\operatorname{logit}(\hat{\pi}))^{2}}}
$$




\section{Example: ICU Data}

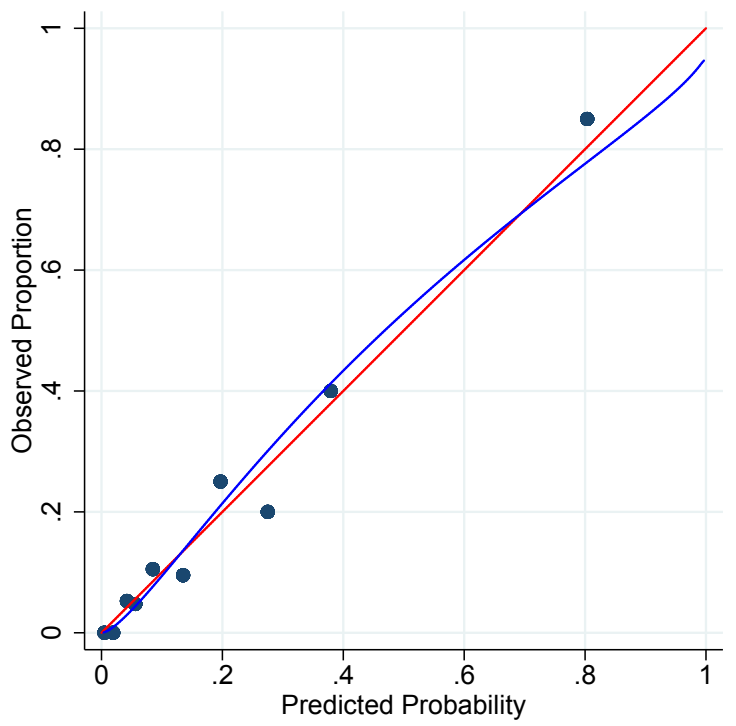




\section{A Goodness of Fit Test}

- When $m$ is selected, we can design a goodness of fit test on

$$
\operatorname{logit}\{P(Y=1)\}=\alpha_{0}+\alpha_{1} \hat{g}+\alpha_{2} \hat{g}^{2}+\ldots+\alpha_{m} \hat{g}^{m} \text {. }
$$

- If perfect fit: $\alpha_{1}=1, \alpha_{0}=\alpha_{2}=\ldots=\alpha_{m}=0$.

- A likelihood ratio test can be used to test the hypothesis

$$
H_{0}: \alpha_{1}=1, \alpha_{0}=\alpha_{2}=\ldots=\alpha_{m}=0
$$

- The distribution of the statistic must account for the forward selection on the same data.

- Inverting the test allows to generate a confidence region around the calibration curve: the calibration belt.

Nattino, G., Finazzi, S., Bertolini, G. (2016). A new test and graphical tool to assess the goodness of fit of logistic regression models. Statistics in medicine. 


\section{Example: ICU Data}

. calibrationbelt

\section{GiViTI Calibration Belt}

Calibration belt and test for internal validation:

the calibration is evaluated on the training sample.

Sample size: 200

Polynomial degree: 2

Test statistic: 1.08

p-value: 0.2994

- estat gof, group(10)

Logistic model for sta, goodness-of-fit test

(Table collapsed on quantiles of estimated probabilities)

number of observations $=200$

number of groups $=10$

Hosmer-Lemeshow $\operatorname{chi2}(8)=\quad 4.00$

Prob $>$ chi2 $=\quad 0.8570$

Nattino, G., Lemeshow, S., Phillips, G., Finazzi, S., Bertolini, G. (2017). Assessing the calibration of dichotomous outcome models with the calibration belt. Stata Journal 


\section{Example: ICU Data}

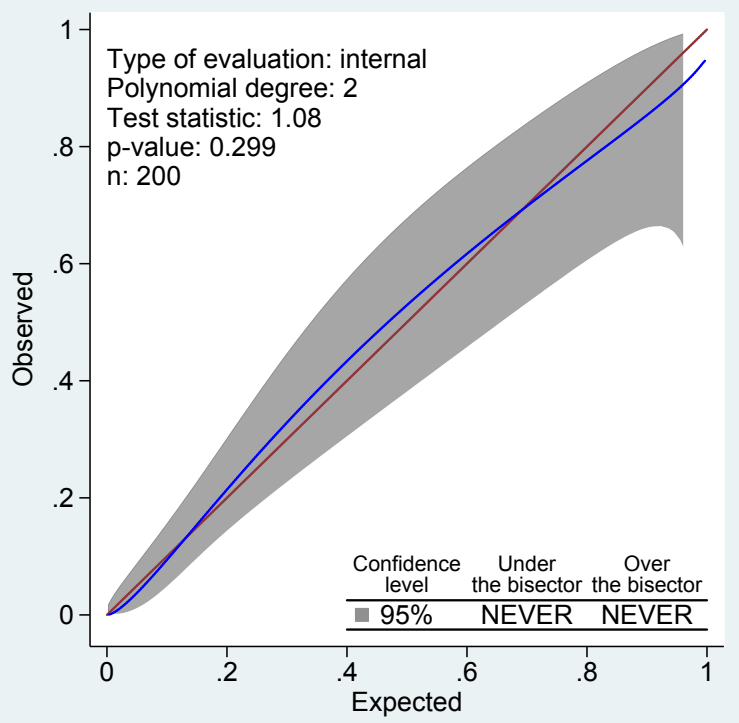




\section{Example 2: Poorly Fitting Model}

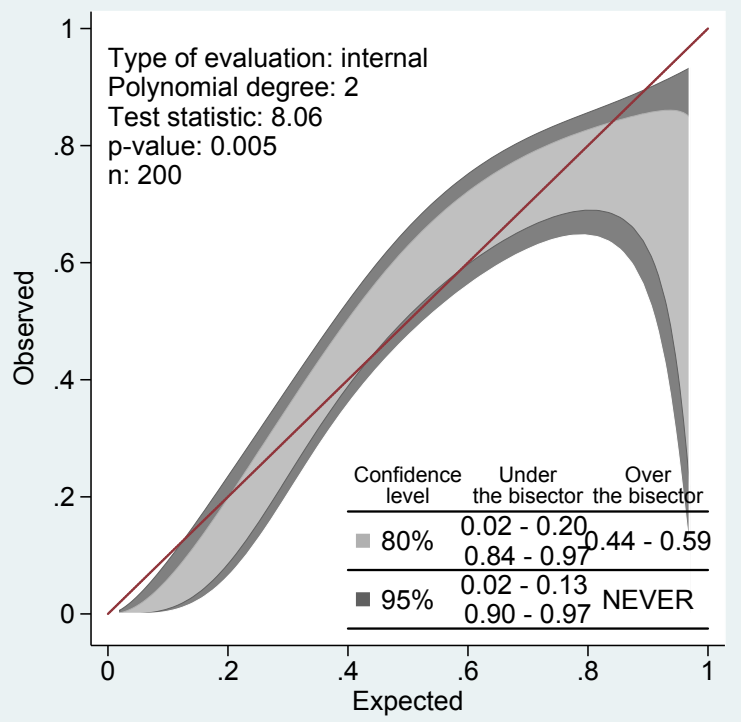




\section{Example 3: External Validation}

- calibrationbelt y phat, devel("external")

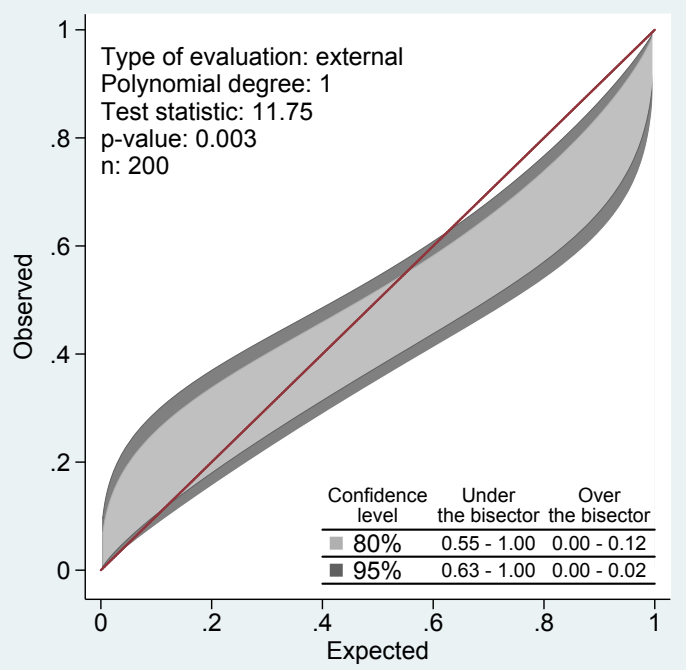




\section{Example 3: External Validation}

. calibrationbelt y phat, cLevel1(.99) cLevel2(.6) devel("external")

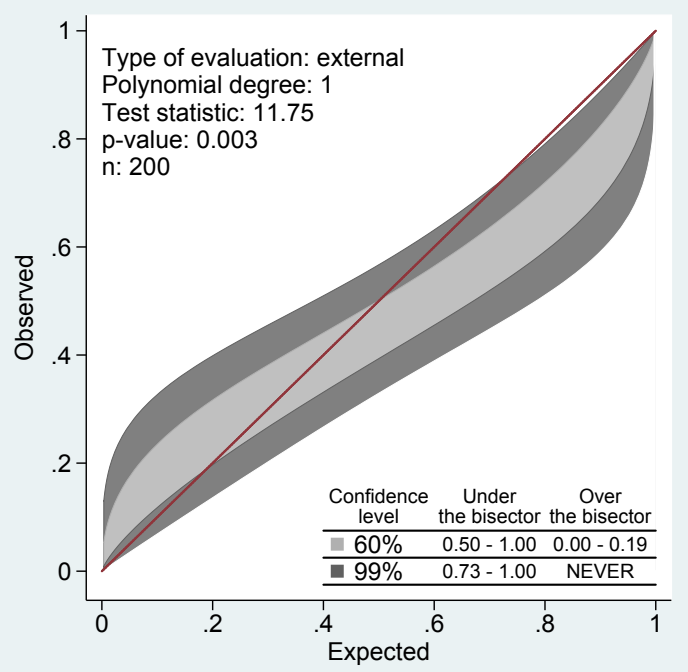




\section{Example 4: Goodness of Fit and Large Samples}

. calibrationbelt

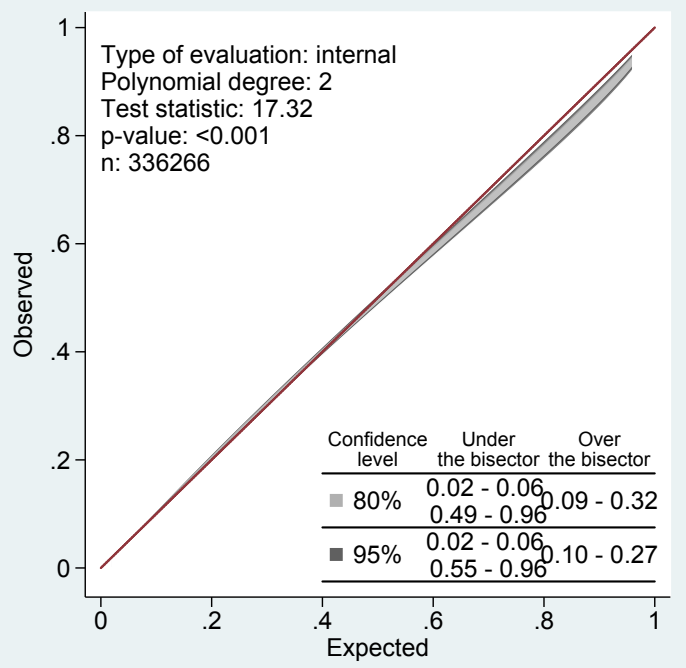




\section{Discussion}

- The calibrationbelt command implements the calibration belt and the related test in Stata.

- Limitation:

- Assumed polynomial relationship.

- Advantages:

- No need of data grouping.

- Informative tool to spot significance of deviations.

- Future work: goodness of fit in very large samples. 